\title{
Theory of the rotation of the Galilean satellites
}

\author{
Benoît Noyelles \\ University of Namur - Dept of Mathematics \\ Rempart de la Vierge 8 - B-5000 Namur - Belgium and \\ IMCCE (Paris Observatory, USTL, UPMC) - CNRS UMR 8028 \\ 77 avenue Denfert-Rochereau - 75014 Paris - France \\ email: noyelles@imcce.fr
}

\begin{abstract}
As most of the natural satellites of the Solar System, the Galilean moons are since a long time assumed to be tidally locked in a spin-orbit synchronous resonance. Thanks to the mission Galileo, we now dispose of enough gravity data to perform 3-dimensional theories of the rotation of these satellites, in particular to model the departure from the exact synchronous rotation. We here present such theories depending on the interior model we consider, in highlighting some observable output data. Inverting them will give us information on the internal structure of these bodies.
\end{abstract}

Keywords. planets and satellites: general

\section{Context}

As most of the natural satellites in the Solar System, the Galilean satellites of Jupiter are assumed to be in a synchronous rotation. This corresponds to a stable dynamical equilibrium, that makes the satellites always present the same face to a fictitious Jovian observer. This equilibrium exists because of the asphericity of these bodies, that are in fact ellipsoids.

The gravitational data of these satellites given by the Galileo spacecraft allow us to give first 3-dimensional studies of their rotations. We here present first our analytical formulation of these problems, then our results, before introducing some ways to improve the modelisation.

\section{Analytical study}

The analytical study starts from the following Hamiltonian:

$$
\begin{aligned}
\mathcal{H}= & \underbrace{\frac{n P^{2}}{2}+\frac{n}{8}\left[4 P-\xi_{q}^{2}-\eta_{q}^{2}\right]\left[\frac{\gamma_{1}+\gamma_{2}}{1-\gamma_{1}-\gamma_{2}} \xi_{q}^{2}+\frac{\gamma_{1}-\gamma_{2}}{1-\gamma_{1}+\gamma_{2}} \eta_{q}^{2}\right]}_{\text {Kinetic energy }} \\
& +\underbrace{n\left(\frac{d_{0}}{d}\right)^{3}\left(1+\delta_{s}\left(\frac{d_{0}}{d}\right)^{2}\right)\left[\delta_{1}\left(x^{2}+y^{2}\right)+\delta_{2}\left(x^{2}-y^{2}\right)\right]}_{\text {Jovian perturbation }}
\end{aligned}
$$

with the following canonical variables: 


$$
\begin{array}{ll}
p=l+g+h & P=\frac{G}{n C} \\
r=-h & R=\frac{G-H}{n C}=P(1-\cos K)=2 P \sin ^{2} \frac{K}{2} \\
\xi_{q}=\sqrt{\frac{2 Q}{n C}} \sin q & \eta_{q}=\sqrt{\frac{2 Q}{n C}} \cos q
\end{array}
$$

where $n$ is the satellite's mean orbital motion, $q=-l$, and $Q=G-L=G(1-\cos J)=$ $2 G \sin ^{2} \frac{J}{2}$. The coefficients of the Hamiltonian are defined as follows:

$$
\begin{array}{ll}
\gamma_{1}=J_{2} \frac{M R^{2}}{C} & \delta_{1}=-\frac{3}{2}\left(\frac{n^{*}}{n}\right)^{2} \gamma_{1} \\
\gamma_{2}=2 C_{22} \frac{M R^{2}}{C} & \delta_{2}=-\frac{3}{2}\left(\frac{n^{*}}{n}\right)^{2} \gamma_{2} \\
\delta_{s} & =\frac{5}{2} J_{2 \hbar}\left(\frac{R_{4}}{d_{0}}\right)^{2}
\end{array}
$$

and the angles can be seen on the figure above, reproduced from Henrard (2005). They use 2 sets of Euler angles, the first one $(h, K, g)$ locates the position of the angular momentum in the first frame $\left(\overrightarrow{e_{1}}, \overrightarrow{e_{2}}, \overrightarrow{e_{3}}\right)$, while the second $(g, J, l)$ locates the body frame $\left(\overrightarrow{f_{1}}, \overrightarrow{f_{2}}, \overrightarrow{f_{3}}\right)$ in the second frame tied to the angular momentum.

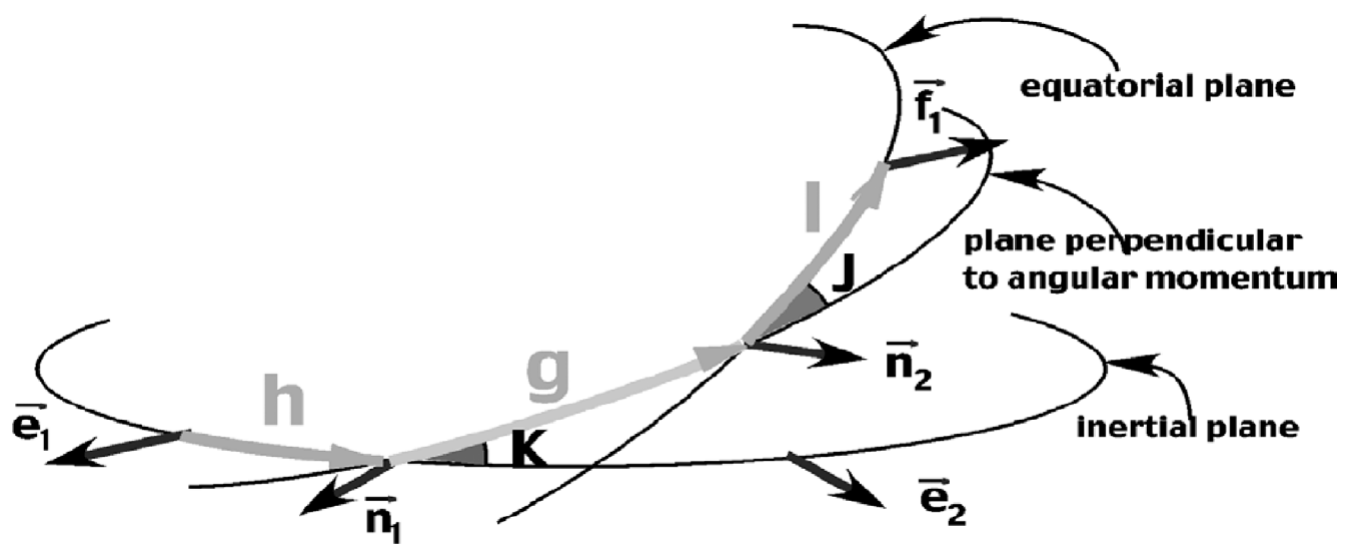

Figure 1. The angles (reproduced from Henrard (2005)).

$x$ and $y$ are the first two coordinates of the center of the perturber (here Jupiter) in the frame $\left(\overrightarrow{f_{1}}, \overrightarrow{f_{2}}, \overrightarrow{f_{3}}\right)$ bound to the satellite. Then we use the model of Henrard \& Schwanen (2004) to obtain the equilibrium $\left(K^{*}, P^{*}\right)$ and the fundamental periods of the 3 proper librations around it: $T_{u}, T_{v}$ and $T_{w}$. At the strict Cassini state, $\sigma=p-\lambda+\pi=0$, $\rho=r+\Omega=0, \xi_{q}=0$ and $\eta_{q}=0$ (i.e. the wobble angle $J$ is null), where $\lambda$ and $\Omega$ are respectively the body's mean longitude and ascending node in an inertial frame.

After some canonical transformations introducing the slow angles, we find the equilibrium of the system, confirming the synchronous rotation and giving the obliquity of the body. Then, a centering of the Hamiltonian on the equilibrium and a conversion into polar coordinates yields:

$$
\mathcal{H}(u, v, w, U, V, W)=\underbrace{\omega_{u} U+\omega_{v} V+\omega_{w} W}_{\text {3-d oscillator }}+\underbrace{\mathcal{P}(u, v, w, U, V, W)}_{\text {Perturbation }},
$$

where $(u, v, w)$ are angles, $(U, V, W)$ the actions associated, and $\left(\omega_{u}, \omega_{v}, \omega_{w}\right)$ the 3 frequencies of the small oscillations about the equilibrium. These oscillations are expected to be damped, but knowing them gives information on the response of the system to the perturbation. The perturbation comes from the non-spherical motion of the studied 
body, and is given by L1 ephemerides (Lainey et al. (2006)). The influence of the perturbations can be used either analytically by way of Lie transforms (cf. Deprit (1969)), or numerically.

\section{Results}

We have shown that the equilibrium obliquity of the body with respect to the normal of the orbital plane is:

$$
\epsilon \approx \frac{\dot{\delta} I}{n\left(\delta_{1}+\delta_{2}+\dot{\delta} / n\right)},
$$

where $\dot{\Omega}$ is the precessional rate of the ascending node of the body, $I$ its orbital inclination, and $n$ its mean frequency, i.e. its spin rate in the case of the synchronous rotation. The proper periods are given in Tab. 1.

Table 1. Proper periods. $T$ is the spin / orbital period, given by Lainey et al. (2006). The 3 periods of the librations of the rotation, respectively in longitude $\left(T_{u}\right)$, latitude $\left(T_{v}\right)$ and wobble $\left(T_{w}\right)$, come from Henrard (2005), Henrard (2005c), Noyelles (2008) and Noyelles (2009).

\begin{tabular}{lrrrr}
\hline & $T$ & $T_{u}$ & $T_{v}$ & $T_{w}$ \\
\hline Io & $1.769 \mathrm{~d}$ & $13.25 \mathrm{~d}$ & $159.39 \mathrm{~d}$ & $229.85 \mathrm{~d}$ \\
Europa & $3.551 \mathrm{~d}$ & $52.70 \mathrm{~d}$ & $3.60 \mathrm{y}$ & $4.84 \mathrm{y}$ \\
Ganymede & $7.154 \mathrm{~d}$ & $186.37 \mathrm{~d}$ & $23.38 \mathrm{y}$ & $30.08 \mathrm{y}$ \\
Callisto & $16.689 \mathrm{~d}$ & $2.46 \mathrm{y}$ & $203.58 \mathrm{y}$ & $317.11 \mathrm{y}$ \\
\hline
\end{tabular}

A difficulty arises for the obliquity of Callisto (Noyelles 2009 \& Fig. 2), because its significant inclination, due to the Solar perturbation, induced what seems to be a chaotic behavior of the orientation of the angular momentum. In fact, it is just a geometrical effect. The Jovian equator at a given date is just an inadequate choice of inertial reference plane, the Laplace Plane has to be considered, which minimizes the variations of the orbital inclination. For most of the natural satellites, it is close enough to the planet's equator, while for Callisto its inclination is $\approx 0.2^{\circ}$.
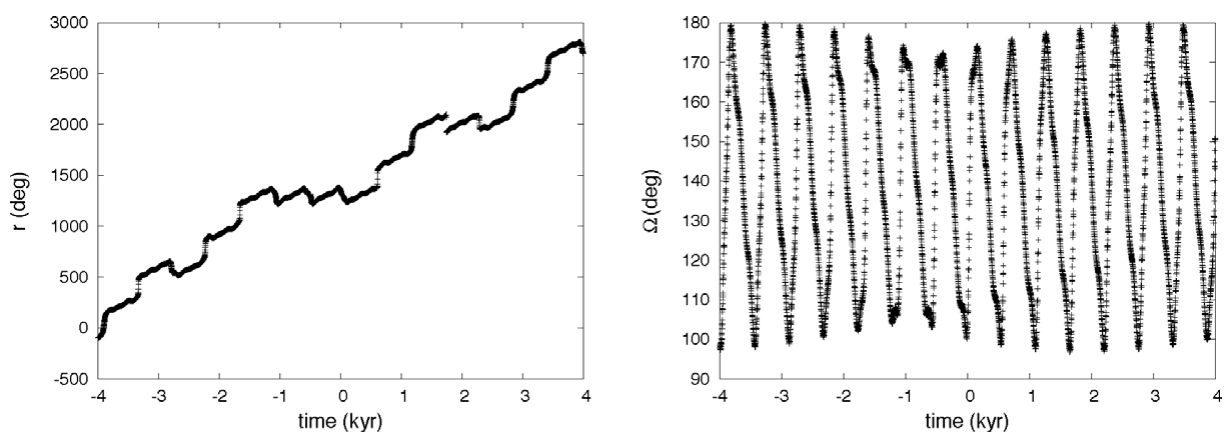

Figure 2. The problem with the inertial reference plane of Callisto. The rotational node of Callisto (left) and the orbital one (right) should have the same mean precession rate. This is in fact a geometrical effect, due to an improper choice of reference plane.

An interesting output variable to look at is the longitudinal motion, that can be seen in longitudinal libration of the body about the synchronous rotation or about the planetsatellite direction, or in the L.O.D. (length of day) (see Fig. 3). This departure from the 
exact synchronous rotation is due to the orbital eccentricity, i.e. to the variations of the planet-satellite distance.
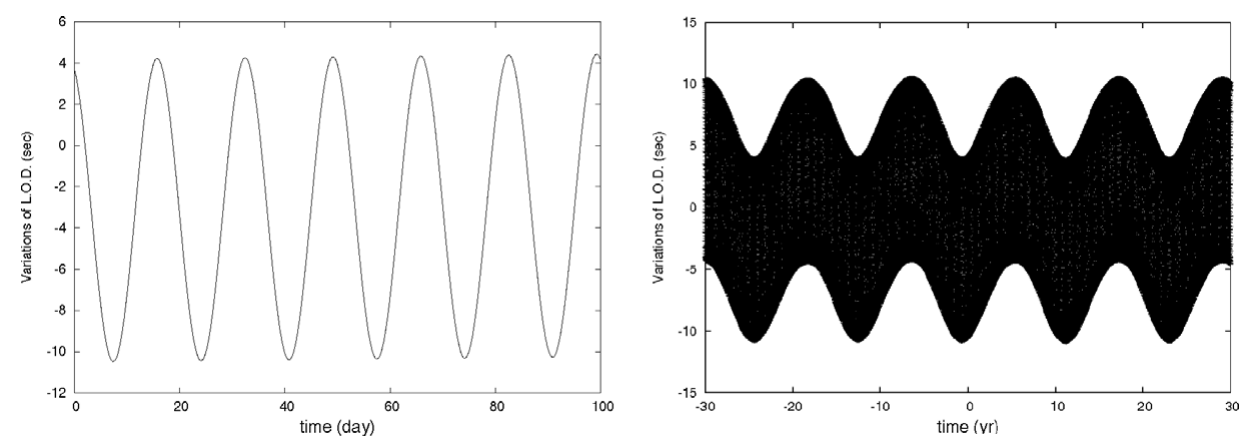

Figure 3. Variations of the Length-Of-Day of Callisto, the mean value being 16.686 days. We can see variations in 16.686 days, i.e. the orbital period of Callisto. Over a larger timescale, we can see a 11.86-yr modulation, due to the Solar perturbation.

\section{Introducing a fluid core}

Considering these satellites as rigid bodies is a first approximation. They are in fact differenciated, multi-layered bodies (perhaps with the exception of Callisto), that have a liquid core (like Io) or an internal ocean (like Europa). So, the next step is to introduce a multi-layered internal structure.

The easiest way to introduce a fluid core is to consider it as spherical. This way, we have no core-mantle interaction and we can just remove the core in the equation, considering the body as an empty body.

Henrard (2008) proposed to consider Io as a body composed of a rigid mantle and a ellipsoidal cavity filled with an inviscid, incompressible and non viscuous fluid. This way, we get core-mantle interactions that should be included in the kinetic energy, i.e.:

$$
\begin{aligned}
\mathcal{T}= & \frac{n}{2(1-\delta)}\left[P^{2}+2 P-2(1+P-Q)\left(\delta+P_{c}-Q_{c}\right)+\left(P_{c}^{2}+2 P_{c}\right) / \delta\right. \\
& \left.+2 \sqrt{Q Q_{c}(2+2 P-Q)\left(2 \delta+2 P_{c}-Q_{c}\right)} \cos \left(q+q_{c}\right)\right] \\
& +\frac{n \epsilon_{1}}{2(1-\delta)}\left[(2+2 P-Q) Q+\left(2 \delta+2 P_{c}-Q_{c}\right) Q_{c} / \delta\right. \\
& \left.+2 \sqrt{Q Q_{c}(2+2 P-Q)\left(2 \delta+2 P_{c}-Q_{c}\right)} \cos \left(q+q_{c}\right)\right] \\
& -\frac{n \epsilon_{2}}{2(1-\delta)}\left[(2+2 P-Q) Q \cos 2 q+\left(2 \delta+2 P_{c}-Q_{c}\right)\left(Q_{c} / \delta\right) \cos q_{c}\right. \\
& \left.+2 \sqrt{Q Q_{c}(2+2 P-Q)\left(2 \delta+2 P_{c}-Q_{c}\right)} \cos \left(q-q_{c}\right)\right],
\end{aligned}
$$

where the subscript $c$ refers to the core, $\delta$ to its size, and $\epsilon_{1}, \epsilon_{2}$ to its shape. Here, a $4^{t h}$ degree of freedom is added, related to the motion of the liquid filling the cavity. The proper frequency associated is close to the spin frequency.

The most spectacular observational consequence is in the amplitude of the forced longitudinal librations. It can be shown that it depends on the size of the core $\delta$. Hence, an observation of these librations can be inverted to measure the size of the core, as has been done for Mercury (Margot et al. (2007)). 


\section{Conclusion}

This contribution presents the elaboration of models of the rotation of the Galilean satellites in 3 degrees of freedom, i.e. without neglecting the obliquity of the polar motion. These theories have been developed both with analytical and numerical tools, with good agreements.

The consideration of realistic internal structures is in progress. This study will need to consider each satellite as individuals, because their interiors are not necessarily similar. We hope that it will be possible to invert the rotation to get clues on their internal structure.

\section{References}

Deprit, A. 1969, Celestial Mechanics, 1, 12

Henrard, J. \& Schwanen, G. 2004, CMEDA, 89, 181

Henrard, J. 2005, Icarus, 178, 144

Henrard, J. 2005b, CMEDA, 91, 131

Henrard, J. 2005c, CMESAA, 93, 101

Henrard, J. 2008, CMESA, 101, 1

Lainey, V., Duriez, L., \& Vienne, A. 2006, A\& $A$, 456, 783

Margot, J.-L., Peale, S. J., Jurgens, R. F. et al. 2007, Science, 316, 710

Noyelles, B. 2008, CMEDA, 101, 13

Noyelles, B. 2009, Icarus, 202, 225 